RAD-16-34

\title{
A DYNAMIC HEAT TRANSFER MODEL TO INVESTIGATE THE EFFECT OF APERTURE SIZE ON THE TEMPERATURE OF A SOLAR RECEIVER
}

\author{
Hamed Abedini Najafabadi and Nesrin Ozalp \\ Mechanical Engineering Department \\ KU Leuven, 3001 Leuven, Belgium
}

\begin{abstract}
Maintaining semi-constant high temperatures inside a solar reactor is a challenge because of the transient nature of the incident solar radiation. For fixed aperture size reactors, changes in incident solar flux directly affect the temperature inside the reactor because of not compensating the fluctuations in incoming solar energy. The present study deals with the dynamic modeling of a solar receiver to simulate the effect of aperture size on the system behavior during unsteady state conditions. Radiation heat transfer analysis of the receiver is studied via Monte Carlo (MC) ray tracing method. MC ray tracing module is coupled to unsteady energy equation solver to develop a model describing the transient behavior of the system. The effect of aperture size on the cavity temperature profile as well as the outlet gas temperature has been investigated and the results confirmed that wide range of temperatures is achievable by changing the aperture size. The paper also demonstrates a thorough comparison on the effectiveness of temperature control via change of aperture size versus via change of gas flow rate. The results show that the change in aperture size can influence the system dynamics more effective than the change in gas flow rate.
\end{abstract}

\section{INTRODUCTION}

Solar thermochemical processes offers promising alternative to traditional combustion based thermochemical reactions with an added value of significantly reduced emission footprint [1]. The basic principle of solar thermochemical processes is the use of concentrated solar energy as high temperature process heat where the radiation enters into the reaction chamber through a small opening, so called an "aperture". Reactants inside a solar reactor absorb energy via convection and radiation. Solar energy is used to raise the reactants' temperature to the necessary dissociation temperature and start the intensive endothermic process. In this manner, solar radiation is converted into chemical energy resulting in a fuel with a higher calorific value. Some examples of thermochemical process are thermal decomposition of limestone [2], the thermal reduction of metal oxides [3], the thermal cracking of natural gas [4], and the thermal gasification of carbonaceous materials [5].

In spite of the aforementioned advantages of solar energy, it has a major drawback due to its inherent nature from sunrise to sunset which instabilities the process dynamics [6]. By using a "cavity-type solar receiver", it is possible to reduce the intrinsic losses in energy conversion efficiency which occurs as a result of re-radiation losses. A cavity type solar receiver is basically a well-insulated enclosure designed to capture incident solar radiation effectively by allowing entry of radiation through a small opening called aperture, so that the intrinsic losses which are proportional to the re-radiation area would be reduced. However, adverse effects of the fluctuating solar radiation creates dynamic instability and leads to lower process efficiencies. Therefore, it is important to develop control strategies capable of handling the constrained non-linear reactor dynamics to achieve higher efficiencies and better use of the available solar energy. 
Despite the essential need to model the dynamics of solar reactors, studies found in literature are limited. One of the first studies on the control of a solar reactor was done by Petrasch and Steinfeld [6] where a set of lumped-parameter reservoirs for mass and energy is incorporated in the model in order to predict the dynamical behavior of a steam/methane-reforming reactor. Another study done by the same group [7] formulates a general non-linear dynamic model that is applicable to a wide range of solar chemical reactors and introduces a process controller to ensure a stable and efficient operation of the system. Another example pioneering work was done by Charvin et al. for metal oxide reduction where a dynamic model of a solar reactor was developed to simulate reactor behavior during transient periods [8]. . On the other hand, Saade et al. employed unsteady mass and energy balances to develop a simplified dynamic model for a solar thermal transport tube reactor [9]. As a follow up study, they developed a Model Predictive Control (MPC) strategy to regulate the solar reactor housing carbon steam gasification process [10]. Recently, Li et al. presented a transient 3D model for hydrogen and carbon monoxide production in a solar reactor by coupling Monte Carlo ray tracing and Lattice Boltzmann (LB) model [11]. Another notable recent study done by Zapata et al. focused on the estimation of outlet temperature based on a control oriented model for a mono tube cavity receiver [12]. It should be note that all of the aforementioned mentioned studies employ a fixed aperture size and they adjust gas flow rate to compensate for fluctuations in incoming solar energy in an effort to maintain stable temperature. Adjustment of flowrate and focus/defocus of heliostats are the most widely used techniques to accommodate changes in incoming radiation. Change of aperture size is a promising alternative specifically for cases where certain flow pattern and flow dynamics should be preserved inside a solar reactor such as in the case of solar methane cracking.

In one of the previous studies done by our research group, a variable size aperture inspired by the human eye was proposed and a thorough heat transfer model was developed for steady state conditions in one dimensional domain [13]. The present work is an improvement to that study by involving transient behavior in the model and integrating the dynamic aspect of the process. The main objective of the present work is to assess the effect of change in gas flow rate versus change in aperture size on receiver temperature

\section{METHODOLOGY}

Schematic of the solar receiver studied in present numerical analysis is shown in Figure 1 . The receiver is made of stainless steel 316L and it consists of a cylindrical cavity with the length of $\mathrm{L}=200 \mathrm{~mm}$, inner radius of $\mathrm{R}_{\text {in }}=60 \mathrm{~mm}$, and outer radius of $\mathrm{R}_{\text {out }}=75 \mathrm{~mm}$. The front wall contains the circular aperture of radius $R_{a p}$, positioned at the second focal plane of the reflector.

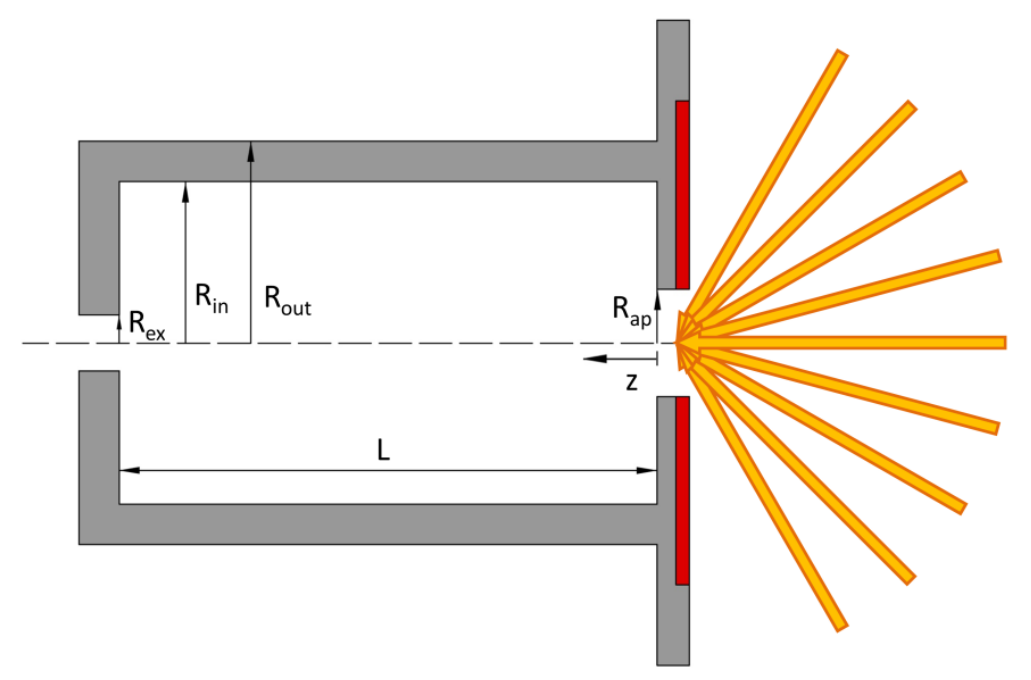

Figure 1. Schematic diagram of the cross section of the solar cavity receiver analyzed 
The air enters the reactor with flow rate of $10 \mathrm{~L} / \mathrm{min}$ (which is equal to $1.936 \times 10^{-4} \mathrm{~kg} / \mathrm{s}$ ) and leaves the reactor through the exit port at the back plate with radius of $R_{e x}=10.45 \mathrm{~mm}$. Exterior surfaces of the receiver are assumed to be perfectly insulated and the interior surfaces of the cavity are assumed to be diffuse gray. Air is modeled as a nonparticipating medium. Referring to Howell et al., monoatomic and diatomic gasses can be treated as non-participating medium which applies to air because it is mainly composed of nitrogen and oxygen [14]. It is also assumed that the radiation properties are independent of temperature.

In simulations, normal radiation ( $I$ ) from the sun was assumed constant. Solar furnace configuration of Paul Scherrer Institute (PSI) was used to calculate the incoming flux for different aperture sizes [15].Monte Carlo (MC) and heat transfer coupled numerical model was used to simulate the thermal behavior of the solar receiver. The model was adapted from the previous work of Usman and Ozalp [13], and modified to account temperature change by time. The inner surfaces are split to several ring elements of equal width. Energy balance for each of these elements in the cavity wall, front plate and back plate elements is given per following equations:

$$
\begin{aligned}
& C_{p, s} m_{c} \frac{d T_{c, i}}{d t}=Q_{a b, c, i}-Q_{e m, c, i}-Q_{c o n v, c-g, i} \\
& C_{p, s} m_{f p, i} \frac{d T_{f p, i}}{d t}=Q_{a b, f p, i}-Q_{e m, f p, i} \\
& C_{p, s} m_{b p, i} \frac{d T_{b p, i}}{d t}=Q_{a b, b p, i}-Q_{e m, b p, i}
\end{aligned}
$$

where $C_{p, s}$ is specific heat capacity of stainless steel. Subscripts $c, f p$ and $b p$ stands for cavity, front plate and back plate, respectively. Subscript $i$ refers to the element number in $c, f p$ or $b p$. $m$ is mass of the element, $T$ is the temperature of the element, $Q_{a b}$ is the absorbed radiation, $Q_{e m}$ is the emitted radiation, and $Q_{c o n v, c-g, i}$ is the convection loss from the cavity wall element to the gas phase which can be calculated according to the following equation:

$$
Q_{c o n v, c-g, i}=h_{c-g} A_{c}\left(T_{c, i}-T_{g, i}\right)
$$

where $h_{c-g}$ is the convective heat transfer coefficient calculated based on the methodology described in Usman and Ozalp [13], $A_{c}$ is the internal surface area of the cavity element, and $T_{g}$ is the gas temperature.

Energy balance for gas phase is given as follows:

$$
d Q_{\text {conv }, c-g}=\dot{m}_{g} C_{p, g} d T_{g}
$$

where $\dot{m}_{g}$ is the mass flow rate of gas air, and $C_{p, g}$ is the specific heat capacity of air. The boundary condition for this equation is:

$$
\left.T_{g}\right|_{z=0}=T_{\infty}
$$

where $T_{\infty}$ is the ambient temperature. Integrating Eq. (5) yields: 


$$
\int_{0}^{Q_{\text {conv },-c, i, i}} d Q_{\text {conv }, c-g}=\dot{m}_{g} C_{p, g} \int_{T_{g, i}}^{T_{g, i+1}} d T_{g}
$$

Reformulating Eq. (7) gives the following equation:

$$
T_{g, i+1}=Q_{c o n v, c-g, i} / \dot{m}_{g} C_{p, g}+T_{g, i}
$$

Based on the boundary conditions, the inlet gas temperature is $300 \mathrm{~K}$ at node $i=1(z=0)$. Gas temperature at each subsequent nodes are calculated from Eqs. (4) and (8).

MC ray tracing method was used to calculate the absorbed radiation by each element. In this method, incident radiation is divided into primary rays from the incoming solar radiation and secondary rays due to emission from the cavity walls. Each primary ray contains the same amount of energy which is calculated as follows:

$$
Q^{p}=Q_{a p} / N_{\text {rays }}^{p}
$$

where $N_{\text {rays }}^{p}$ is the number of primary rays, and $Q_{a p}$ is the total solar energy intercepted by the aperture. The energy of each of secondary rays is calculated according to the following equation:

$$
Q^{s}=Q_{\text {em }} / N_{\text {rays }}^{s}
$$

where $N_{\text {rays }}^{s}$ is the number of secondary rays, and $Q_{e m}$ is the energy emitted by the cavity, front plate or back plate accordingly, which is calculated as follows:

$Q_{e m}=A_{x} \varepsilon \sigma T_{x}^{4}$

where $A$ is the area and the subscript $x$ represents the cavity, front plate or back plate accordingly. The emissivity is denoted by $\varepsilon$, and $\sigma$ is the Stefan Boltzmann constant $\left(5.67 \times 10^{-8} \mathrm{Wm}^{-2} \mathrm{~K}^{-4}\right)$.

After intersection of the primary and secondary rays with the cavity walls, the probability of reflection or absorption is calculated using a random number $R$ between 0 and 1 . If $R \leq \alpha$, the ray is absorbed, whereas if $R>\alpha$, the ray is reflected. $\alpha$ is the absorptivity which is equal to $\varepsilon=0.8$ per Kirchhoff's law for diffuse gray surfaces. If the ray is absorbed, the count is recorded, and its history is terminated. If the ray is reflected, there would be two cases based on the reflective configuration of the inner walls. For specular reflection, the direction of reflected ray is estimated from:

$\hat{r}=\hat{u}-2(\hat{n} \cdot \hat{u}) \hat{n}$

where $\hat{u}$ is the normal vector of the intersected ray, and $\hat{n}$ is the normal unit vector of the surface at the point of reflection. For diffusive reflection, the direction of reflected ray is estimated from the cone and polar angles that the emitted ray makes with the surface of intersection which are given by:

$$
\begin{aligned}
& \beta=\sin ^{-1}\left(R_{1}\right) \\
& \varphi=2 \pi R_{2}
\end{aligned}
$$


where $R_{1}$ and $R_{2}$ are the random numbers between 0 and 1 . All rays are traced according to this procedure until they are either absorbed by the inner surfaces of the reactor or escaped the cavity through the aperture or the exit port.

After tracing all of the rays, the absorbed radiation by a surface element $i$ is calculated from:

$Q_{a b, i}=n_{a b, i}^{p} Q_{p}+\sum_{j=1}^{N} n_{a b, i, j}^{s} Q_{s, j}$

where $n_{a b, i}^{p}$ is the number of primary rays absorbed by element $i, n_{a b, i, j}^{s}$ is the number of secondary rays emitted by element $j$ and absorbed by element $i$.

Once the absorption terms are found from MC ray tracing method, Eq. (1) to (4) and (8) can be solved to obtain dynamic temperature profile of the system. Total number of 600,000 primary rays, and 900,000 secondary rays were used in this simulation. Each cavity wall, front plate and back plate are split to 100 elements which lead to 300 differential equations and 100 algebraic equations. Solution to these algebraic-differential equations (ADE) was made using Runge-Kutta 13 order method.

\section{RESULTS AND DISCUSSION}

\subsection{Model verification}

To verify the MC ray tracing code, the apparent absorptivity was determined as a function of surface absorptivity and compared with those reported in Ref. [16]. Apparent absorptivity was calculated by dividing the number of rays absorbed by the inner cavity walls to the number of primary rays entering the receiver. The solar reactor in Ref. [16] has the following dimensions: $L=263 \mathrm{~mm}, R_{\text {in }}=55 \mathrm{~mm}, R_{\text {out }}=150 \mathrm{~mm}$, and $R_{e x}=0$. Figure 2 shows the apparent absorptivity as a function of surface absorptivity for the baseline configuration for diffuse or specular reflective inner walls, and for the aperture radiuses of 20,30 , and $40 \mathrm{~mm}$. As it is seen in this figure, there is a good agreement between our results and those reported in Ref. [16].

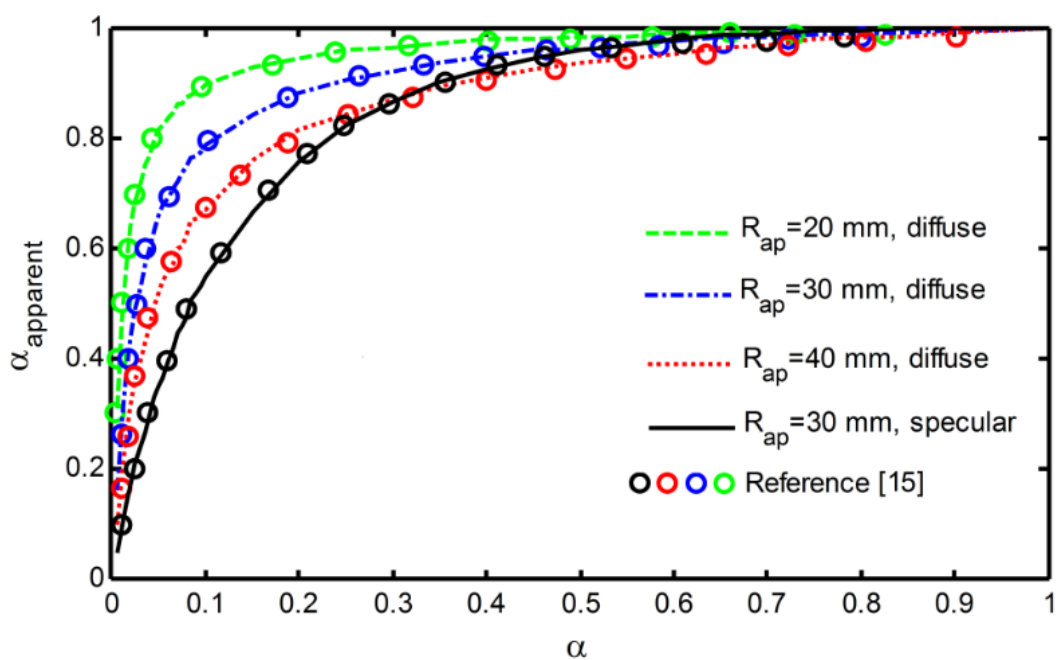

Figure 2. Apparent absorptivity as a function of surface absorptivity for the baseline configuration for diffuse or specular reflective inner walls, and for different aperture sizes

As the second step of the verification, the stagnation temperature distribution along the cavity wall was calculated for the same geometry using our code and then was compared with results given in Ref. [13]. Stagnation temperature is defined as the highest possible temperature that the receiver would attain at equilibrium in the absence of energy losses. To calculate the stagnation temperature, the $Q_{c o n v, c-g, i}$ in Eq. (1) was set to zero, and the time was set to $24 \mathrm{~h}$ to let the system reach steady 
state. The results of this comparison are shown in Figure 3, which shows an agreement with the results given in Ref. [13].

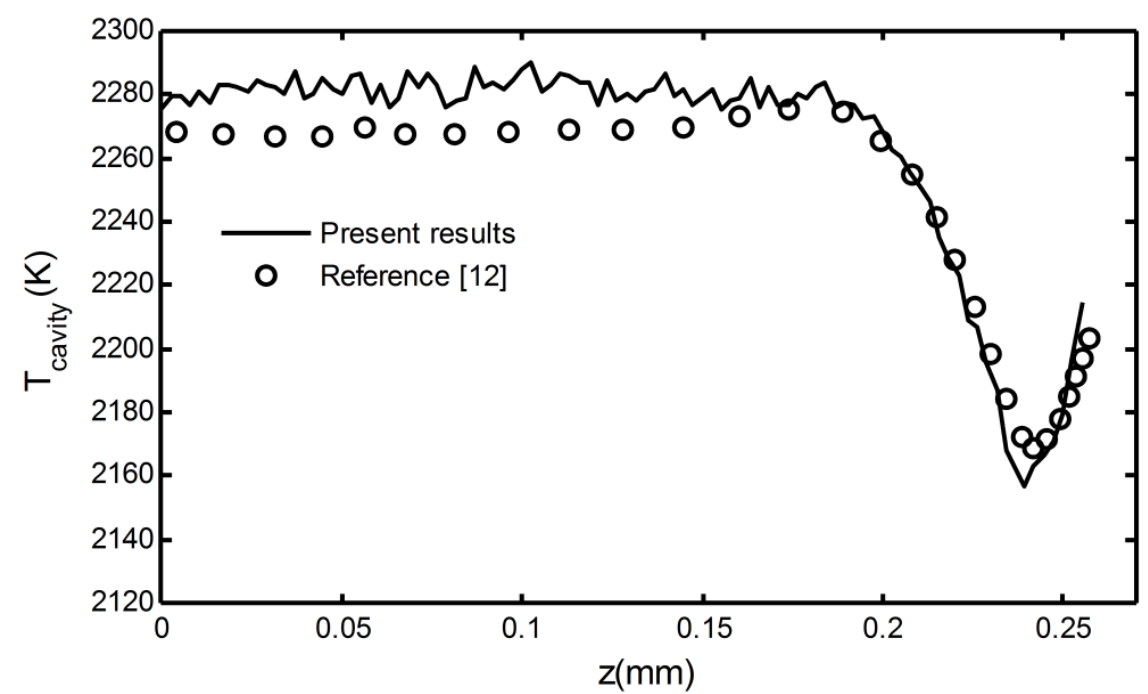

Figure 3. Steady state stagnation temperature in cavity walls $\left(\mathrm{z}^{*}=\mathrm{L}-\mathrm{z}\right)$

\subsection{Dynamic temperature profile inside the solar receiver}

The results of the variation in temperature versus time at different points of cavity wall $(\zeta=z / L)$ are shown in Figure 4a. Dynamic temperature variation of the outlet gas, front plate and back plate are also in Figure 4b. The aperture size in these simulations is $30 \mathrm{~mm}$. It is seen that the dynamic temperature variation is different in cavity wall, front plate and back plate.
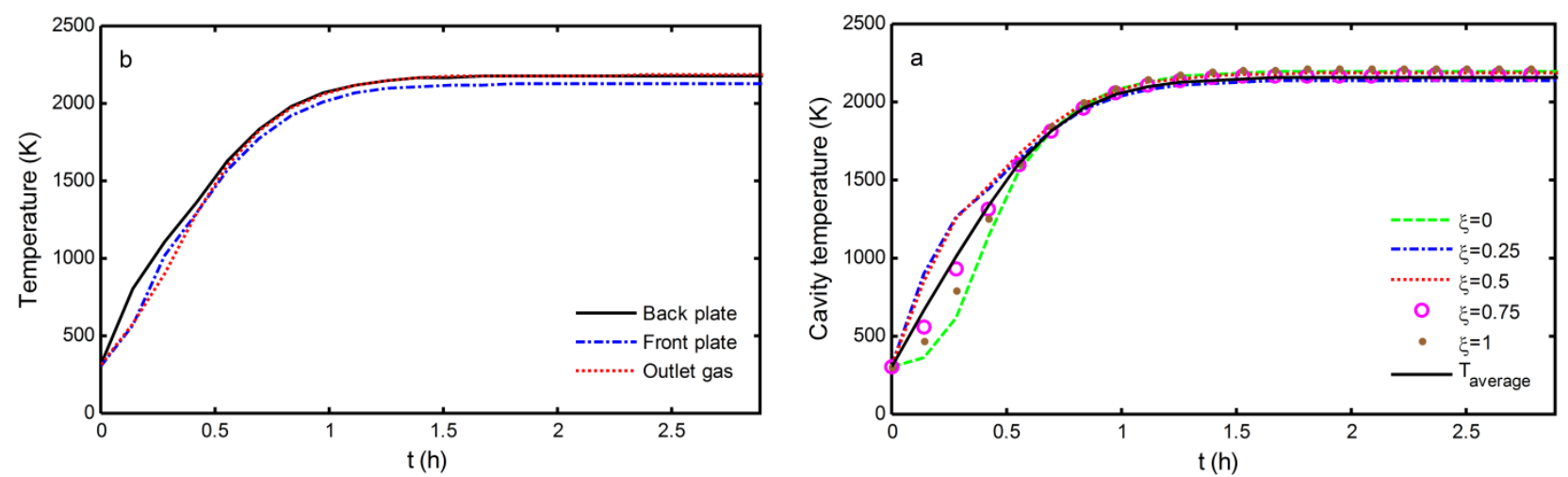

Figure 4. Dynamic variation of the temperature at (a) cavity wall, (b) front plate, back plate and outlet gas

\subsection{Effect of aperture size on the outlet gas temperature}

The effect of aperture size on the dynamics of outlet gas temperature is shown in Figure 5. The results indicate that by changing the aperture size; a wide range of temperature can be obtained which confirms the suitability of using this parameter in the control of the system. 


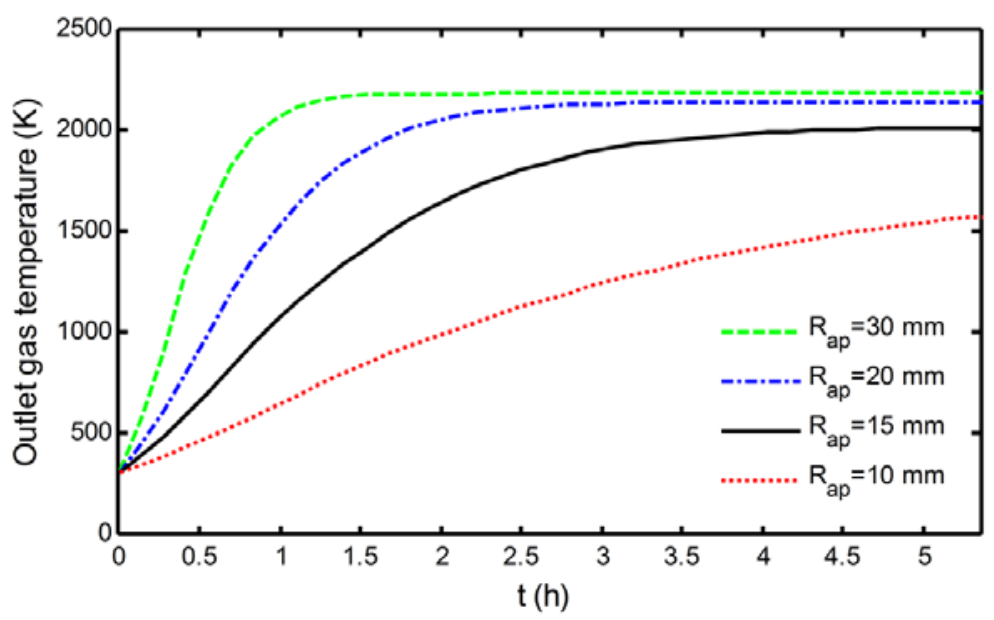

Figure 5. Effect of aperture size on the dynamics of the outlet gas temperature

\subsection{Effect of aperture size versus gas flow rate}

The most common technique practiced to control reactor or receiver temperature is the adjustment of gas flow rate. Change of aperture size is a promising alternative for cases where the flow dynamics must be preserved. In order to assess the effectiveness of these two methods, the dynamic response of the system versus equal step changes in these two parameters can be examined. Figure 6 shows the effect of one step increase in aperture size and gas flow rate on the outlet gas temperature. In these simulations, the aperture radius was increased from its initial value of $30 \mathrm{~mm}$ by $30 \%$, and the gas flow rate was increased from its initial value of $1.936 \times 10^{-4} \mathrm{~kg} / \mathrm{s}$ by $30 \%$. As it is seen, the effect of aperture size on the outlet temperature is larger than that of the mass flow rate.

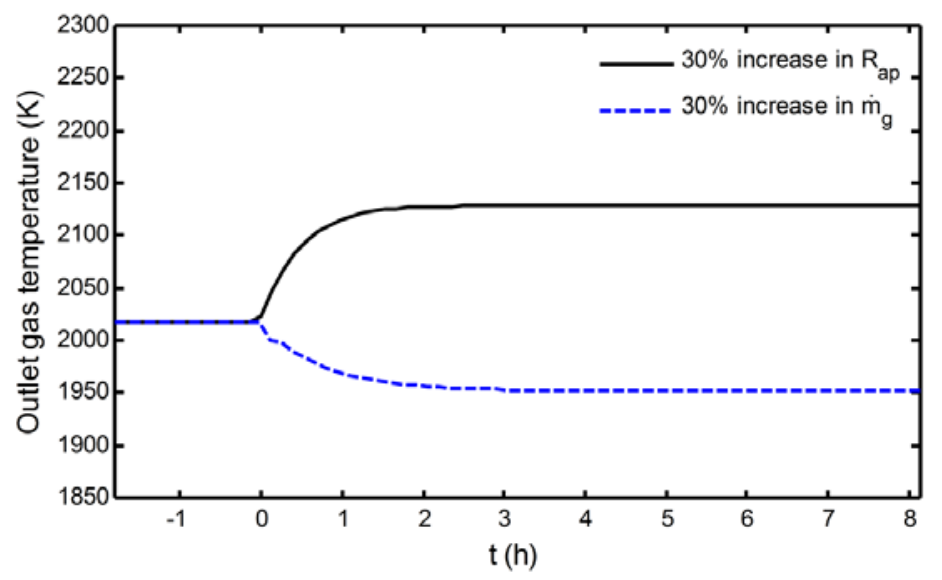

Figure 6. Effect of one step increase in aperture size and gas flow rate on the outlet gas temperature

To have a better comparison, a first order transfer function was fitted to these dynamic responses according to the method described by Smith and Corripio [17]. The mathematical formula for this first order transfer function is as follows:

$$
\Delta y / \Delta \bar{u}=k(1-\exp (-t / \tau))
$$

where $y$ is the system output (outlet gas temperature), $\bar{u}$ is the normalized parameter (aperture size or gas flow rate), $k$ and $\tau$ are the parameters of the first order transfer function. The values of $k$ and $\tau$ for these step responses are tabulated in Table 1 . The absolute value of $k$ for aperture size change is more significant than the mass flow rate change, which indicates that the effect of aperture size on the final temperature is bigger. Furthermore, the $\tau$ value for aperture size change is smaller than the 
gas flow rate change, which indicates that the aperture size influences the process with faster dynamics.

Table 1. Parameters of the first order transfer function in response to a step change in aperture size and gas flow rate

\begin{tabular}{lcc}
\hline Variable & $k$ & $T$ \\
\cline { 2 - 3 } Aperture size & 376.67 & 0.4541 \\
Gas flow rate & -220.67 & 0.7458 \\
\hline
\end{tabular}

It should be noted that the effect of aperture size on the temperature of the system is nonlinear. As it is seen in Figure 7, change in aperture radius from $15 \mathrm{~mm}$ to $20 \mathrm{~mm}$ yields about $300 \mathrm{~K}$ change in outlet temperature.

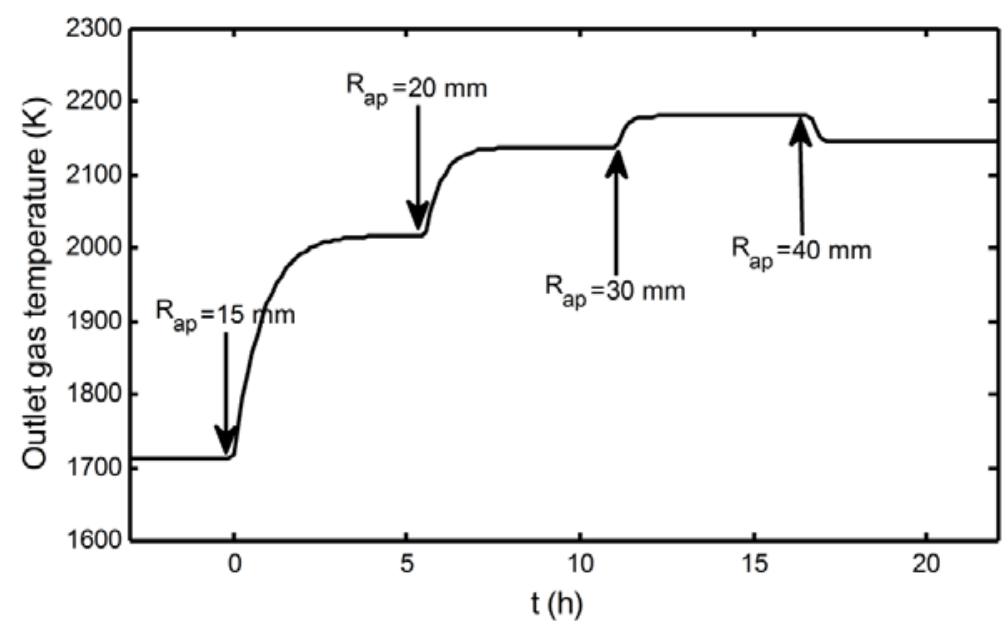

Figure 7. Effect of different step changes in aperture size on the outlet gas temperature

On the other hand, change in aperture radius from $20 \mathrm{~mm}$ to $30 \mathrm{~mm}$ increases the outlet gas temperature by $60 \mathrm{~K}$. When the aperture radius is increased from $30 \mathrm{~mm}$ to $40 \mathrm{~mm}$, the outlet gas temperature decreases.

Table 2. Process gain of the system in response to different step changes in aperture size

\begin{tabular}{ccc}
\hline $\begin{array}{c}\text { Initial value of } R_{a p} \\
(\mathrm{~mm})\end{array}$ & $\begin{array}{c}\text { Final value of } R_{a p} \\
(\mathrm{~mm})\end{array}$ & $\begin{array}{c}\text { Process gain } \\
(\mathrm{K} / \mathrm{mm})\end{array}$ \\
\hline 10 & 15 & 61.00 \\
15 & 20 & 23.96 \\
20 & 30 & 4.29 \\
30 & 40 & -3.59 \\
\hline
\end{tabular}

The process gain $\left(\Delta y /\left.\Delta u\right|_{t=\infty}\right)$ of the system corresponding to these step changes is tabulated in Table 2. These results indicate that when aperture size is changed, the process gain of the system varies significantly which makes the control of this system more challenging.

\section{CONCLUSIONS}

In this study, an algebraic differential equation (ADE) model is proposed to describe the dynamical behavior of a solar receiver. This model was developed by coupling unsteady energy balances of 
the cavity parts and the Monte Carlo (MC) ray tracing method. The model was used to simulate the dynamical behavior of a solar receiver using the PSI solar furnace configuration as the heat source. The simulation results indicate that the aperture size has a significant impact on the cavity temperature profile. The results also indicates that the aperture size can influence the system dynamics more effective than the change in gas flow rate. These findings justify the impact of aperture size as the parameter for the temperature control of a solar receiver. However, because the effect of aperture size on the dynamics of the system is highly non-linear, a robust non-linear controller must be designed.

\section{REFERENCES}

1. N. Ozalp, A. Kogan, M. Epstein, "Solar decomposition of fossil fuels as an option for sustainability”, International Journal of Hydrogen Energy, vol. 34, pp. 710-720, 2009.

2. A. Meier, E. Bonaldi, G. M. Cella, W. Lipinski, "Multitube rotary kiln for the industrial solar production of lime”, Journal of Solar Energy Engineering, vol. 127, pp. 386-395, 2005.

3. L. Xiao, S. Y. Wu, Y. R. Li, “Advances in solar hydrogen production via two-step water-splitting thermochemical cycles based on metal redox reactions", Renewable Energy, vol. 41, pp. 1-12, 2012.

4. D. Hirsch, A. Steinfeld, "Solar hydrogen production by thermal decomposition of natural gas using a vortex-flow reactor”, International Journal of Hydrogen Energy, vol. 29, pp.47-55, 2004.

5. N. Gokon, T. Izawa, T. Abe, T. Kodama, "Steam gasification of coal cokes in an internally circulating fluidized bed of thermal storage material for solar thermochemical processes", International Journal of Hydrogen Energy, vol. 39, pp. 11082-11093, 2014.

6. J. Petrasch, A. Steinfeld, "Dynamics of a solar thermochemical reactor for steam-reforming of methane”, Chemical Engineering Science, vol. 62, pp. 4214-4228, 2007.

7. J. Petrasch, P. Osch, A. Steinfeld, "Dynamics and control of solar thermochemical reactors", Chemical Engineering Journal, vol. 145, pp. 362-370, 2009.

8. P. Charvin, S. Abanades, P. Neveu, F. Lemont, G. Flamant, "Dynamic modeling of a volumetric solar reactor for volatile metal oxide reduction”, Chemical Engineering Research and Design, vol. 86, pp. 1216-1222, 2008.

9. E. Saade, C. Bingham, D. E. Clough, A. W. Weimer, "Dynamics of a solar-thermal transporttube reactor”, Chemical Engineering Journal, vol. 213, pp. 272-285, 2012.

10. E. Saade, D. E. Clough, A. W. Weimer, "Model predictive control of a solar-thermal reactor" Solar Energy, vol. 102, pp. 31-44, 2014.

11. L. Li, C. Chen, A. Singh, N. Rahmatian, N. AuYeung, K. Randhir, R. Mei, J. F. Klausner, D. W. Hahn, J. Petrasch, "A transient heat transfer model for high temperature solar thermochemical reactors”, International Journal of Hydrogen Energy, vol. 41, pp. 2307-2325, 2016.

12. J. I. Zapata, J. Pye, K. Lovegrove, "A transient model for the heat exchange in a solar thermal once through cavity receiver”, Solar Energy, vol. 93, pp. 280-293, 2013.

13. S. Usman, N. Ozalp, "Numerical and optical analysis of solar power level adaptable solar reactor”, Heat Transfer Engineering, vol. 35, pp. 1405-1417, 2014.

14. J. R. Howell, R. Siegel, M. P. Menguc, “Thermal radiation heat transfer”, 5th Ed., CRC Press, New York, 2011.

15. P. Haueter, T. Seitz, A. Steinfeld, “A new high-flux solar furnace for high-temperature thermochemical research”, Journal of Solar Energy Engineering, vol. 121, pp. 77-80, 1999.

16. S. Kräupl, A. Steinfeld, "Monte Carlo radiative transfer modeling of a solar chemical reactor for the co-production of zinc and syngas”, Journal of Solar Energy Engineering, vol. 127, pp. 102-108, 2005.

17. C. A. Smith, A. B. Corripio, "Principles and practices of automatic process control”, 2nd Ed., John Wiley \& Sons, Inc., New York, 1997. 